\title{
Rapid-onset diabetic ketoacidosis secondary to nivolumab therapy
}

\author{
Senhong Lee', Aparna Morgan', Sonali Shah'1 and Peter R Ebeling1,2 \\ 'Department of Endocrinology, Monash Health, Clayton, Victoria, Australia and 2Department of Medicine, School of \\ Clinical Sciences, Monash University, Clayton, Victoria, Australia
}

Correspondence should be addressed

to $S$ Lee

Email

senhonglee@hotmail.com

\section{Summary}

We report a case of a 67-year-old man with type 2 diabetes presented with diabetic ketoacidosis, two weeks after his first dose of nivolumab therapy for non-small-cell lung carcinoma. He was started on empagliflozin two days prior in the setting of hyperglycaemia after the initiation of nivolumab therapy. Laboratory evaluation revealed an undetectable C-peptide and a positive anti-glutamic acid decarboxylase (GAD) antibody. He was treated with intravenous fluids and insulin infusion and was subsequently transitioned to subcutaneous insulin and discharged home. He subsequently has developed likely autoimmune thyroiditis and autoimmune encephalitis.

\section{Learning points:}

- Glycemic surveillance in patients receiving immune checkpoint inhibitors is recommended.

- Early glycemic surveillance after commencement of anti-programmed cell death-1 (PD-1) inhibitors may be indicated in selected populations, including patients with underlying type 2 diabetes mellitus and positive antiglutamic acid decarboxylase (GAD) antibody.

- Sodium-glucose co transporter-2 (SGLT2) inhibitors should be used with caution in patients on immunotherapy.

\section{Background}

The rapid development of diabetic ketoacidosis after a single dose of an immune checkpoint inhibitor is extremely rare. This case highlights the importance of glycemic surveillance in patients receiving immune checkpoint inhibitors. We also highlight the risk factors for rapid development of diabetic ketoacidosis postimmunotherapy, which are not well established in the literature.

\section{Case presentation}

A 67-year-old man with a 40 pack-year smoking history was referred to the respiratory department in October 2016 for investigation of a persistent cough. His medical history was significant for hypertension, hypercholesterolemia, chronic obstructive pulmonary disease and a six-year history of presumed type 2 diabetes mellitus (T2DM) well managed on oral glucose-lowering agents. There was no known family history of diabetes or other autoimmune disease. He underwent bronchoscopy with biopsy of the left main bronchus revealing a diagnosis of squamous cell carcinoma (SCC). Further staging revealed unresectable T4NOM0 non-small-cell lung carcinoma (NSCLC). He subsequently received chemotherapy (6 cycles of weekly carboplatin and paclitaxel from December 2016 to January 2017) and radiotherapy. Follow-up CT chest in July 2017 revealed increased left hilar infiltrates and occlusion of left bronchus, consistent with local recurrence. Repeat bronchoscopic biopsy in August 2017 confirmed a left middle lobe SCC. He was then planned for nivolumab $312 \mathrm{mg}(3 \mathrm{mg} / \mathrm{kg})$ every two weeks, and he received his first dose on the 31st of August 2017. 
Two weeks after the first dose of nivolumab, our patient presented to the chemotherapy day unit for his second dose of nivolumab therapy. A random blood glucose level (BGL) was $28.6 \mathrm{mmol} / \mathrm{L}$ and a ketone level was $7.0 \mathrm{mmol} / \mathrm{L}$. A venous blood gas subsequently revealed a metabolic acidosis with a $\mathrm{pH}$ of 7.0 (7.32-7.42), partial pressure of carbon dioxide $\left(\mathrm{pCO}_{2}\right)$ of $34 \mathrm{mmHg}$ (41-51 mmHg), bicarbonate ( $\left.\mathrm{HCO}^{3-}\right)$ of $8.3 \mathrm{mmol} / \mathrm{L}(21-$ $30 \mathrm{mmol} / \mathrm{L}$ ) with an increased anion gap of $36.7 \mathrm{mEq} / \mathrm{L}$ $(8-16 \mathrm{mEq} / \mathrm{L})$. On further questioning, he described two-day history of lethargy, polyuria and polydipsia associated with elevated BGL readings at home (between 20 and $30 \mathrm{mmol} / \mathrm{L}$ ). In this setting, his local medical officer had commenced empagliflozin $12.5 \mathrm{mg}$ twice a day (BD) two days prior to his presentation as third-line agent for treatment of diabetes in addition to metformin $850 \mathrm{mg} \mathrm{BD}$ and sitagliptin $50 \mathrm{mg}$ BD. A diagnosis of diabetic ketoacidosis (DKA) was made, and patient was transferred to the Emergency Department for further management.

\section{Investigation}

Further laboratory evaluation revealed a C-peptide of $<0.1 \mathrm{ng} / \mathrm{mL} \quad(0.9-7 \mathrm{ng} / \mathrm{mL})$ with a paired BGL of $15 \mathrm{mmol} / \mathrm{L}$. He was found to have elevated anti-GAD antibody (glutamic acid decarboxylase) of $>2000 \mathrm{U} /$ $\mathrm{mL}(<5 \mathrm{U} / \mathrm{mL})$ but was negative for anti-IA2 antibody (tyrosine phosphatase-related islet antigen 2) and anti-ZnT8 antibody (Zinc Transporter 8 antibody). His glycosylated hemoglobin (HbA1c) at presentation was $7.6 \%$. His thyroid-stimulating hormone (TSH) was within the normal range. His septic screen including blood culture, urine culture and chest X-ray was negative.

\section{Treatment}

Given the temporal relationship between the onset of symptoms and initiation of nivolumab therapy, a diagnosis of nivolumab-induced DKA was made. The undetectable C-peptide level and high-titre anti-GAD antibody $(>2000 \mathrm{U} / \mathrm{mL})$ were suggestive of underlying autoimmune diabetes. Unfortunately, in the absence of a pre-nivolumab C-peptide and anti-GAD antibody level, we were unable to establish whether our patient seroconverted prior or during nivolumab therapy. The initiation of empagliflozin, a sodium-glucose cotransporter 2 (SGLT2) two days prior to his DKA may have contributed to his rapid development of DKA.
He was treated with intravenous fluids and insulin infusion and was subsequently transitioned to subcutaneous insulin and discharged home. His case was discussed with the oncology team, and the plan was to continue with nivolumab therapy.

\section{Outcome and follow-up}

Three months post discharge, he remained on insulin for his diabetes. He developed hyperthyroidism, likely secondary to autoimmune thyroiditis. His TSH was $0.07 \mathrm{mU} / \mathrm{L}(0.4-4.8 \mathrm{mU} / \mathrm{L})$, free thyroxine (fT4) was $20.1 \mathrm{pmol} / \mathrm{L}(8-16 \mathrm{pmol} / \mathrm{L})$ and thyroid autoantibodies were negative. Unfortunately, a thyroid uptake scan was unable to be performed due to recent contrast exposure. He also had developed recurrent seizures and was being investigated for suspected autoimmune encephalitis.

\section{Discussion}

Immune checkpoint inhibitors (ICIs) are medications that activate anti-tumor responses by disrupting the inhibitory signaling to T cells (1). Nivolumab is an ICI that selectively blocks the programmed cell death-1 (PD-1) receptors found on the T cells (1). PD1-receptors inhibit kinase signaling pathways that normally lead to T-cell activation when bound to its ligands (PD-L1 and PD-L2) (1). Nivolumab has been approved by the Food and Drug Administration (FDA) for the treatment of multiple cancers - NSCLC, melanoma, renal cell carcinoma, Hodgkin's lymphoma, SCC of head and neck, urothelial carcinoma and colorectal carcinoma (1). These medications can cause immune-related adverse events including colitis, pneumonitis, nephritis, hepatitis, encephalitis and endocrinopathies (1). Immune endocrinopathies include thyroid dysfunction, hypophysitis, adrenal insufficiency and autoimmune diabetes mellitus (1).

Our current knowledge of DKA as a complication of anti-PD-1 antibody therapy is based on case reports and small case series. Most of these patients present in the setting of autoimmune diabetes induced by immunotherapy (2). The development of DKA associated with anti-PD1 antibody treatment typically presents after several doses of immunotherapy and that it is uncommon to develop after a single dose (2). In fact, our patient is only the fourth case of anti-PD1 antibody-induced DKA in the literature, to present within two weeks post treatment initiation $(3,4,5)$. 
Table 1 Summary of patients who presented with DKA within two weeks post anti-PD-1 antibody therapy.

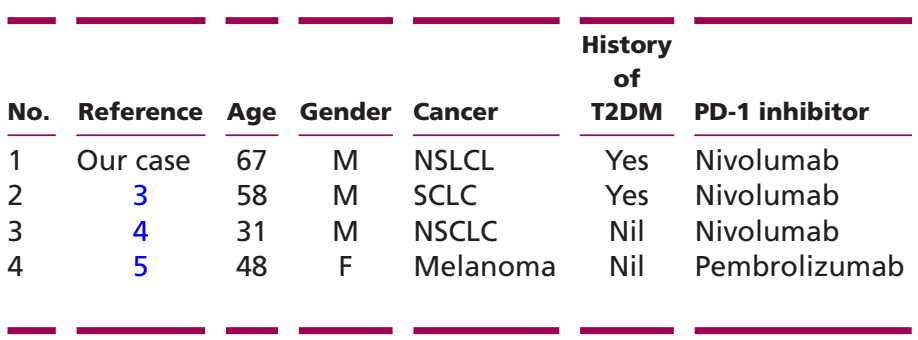

Four patients who presented with DKA within two weeks post anti-PD-1 antibody therapy were studied (Table 1). The mean age of presentation was 51 years (age range 31-67). Interestingly, three out of four of them received nivolumab for lung cancer, while the other one received pembrolizumab for melanoma. Half of the patients had a diagnosis of T2DM prior to receiving anti-PD-1 antibody therapy. However, it is noteworthy that 3 patients out of 4 had an HbA1c of $\geq 6.5 \%$ at presentation that may suggest undiagnosed diabetes prior to receiving pembrolizumab only two weeks earlier (patient 4). It is possible that patients with underlying T2DM present more rapidly with DKA due to their reduced islet beta cell reserve (6). Interestingly, all four patients were positive for anti-GAD antibodies. Gauci et al. identified that patients with anti-GAD antibodies had a more rapid onset of diabetes post-immunotherapy compared with those that were anti-GAD antibody negative; 3 weeks vs 12.5 weeks (2). The significance of anti-GAD positivity among these patients needs to be clarified with larger studies.

It is noteworthy that our patient was started on empagliflozin two days prior to his presentation with DKA. It has been reported in the literature that SGLT2 inhibitors may increase the risk of DKA shortly after their initiation (7). The initiation of empagliflozin may have accelerated the rapid development of DKA in addition to the patient's pre-existing risk factors. This highlights the importance of avoiding SGLT2 in patients who are at risk of developing DKA, including concomitant use of antiPD1-inhibitors.

Our patient also developed likely autoimmune thyroiditis and autoimmune encephalitis. Our patient's autoimmune diabetes may be a risk factor for him to develop other immune-related complications from nivolumab. Therefore, clinicians should remain vigilant in monitoring for other immune-related complications in patients with autoimmune diabetes and vice versa.

DKA needs to be recognized as a rare, but important side effect of anti-PD-1 inhibitors. Early glycemic surveillance after commencement of this therapy is

\begin{tabular}{|c|c|c|c|c|}
\hline $\begin{array}{c}\text { Onset to } \\
\text { presentation } \\
\text { (days) }\end{array}$ & $\begin{array}{l}\text { Glucose } \\
\text { (mmol/L) }\end{array}$ & $\begin{array}{l}\text { C-peptide } \\
\text { (ng/mL) }\end{array}$ & $\begin{array}{c}\text { HbA1c } \\
(\%)\end{array}$ & Antibodies \\
\hline 14 & 28.6 & $<0.10$ & 7.6 & Positive GAD \\
\hline 7 & 39.9 & $<0.10$ & 9.7 & Positive GAD65 \\
\hline 13 & 38.4 & $<0.03$ & 6.4 & Positive GAD \\
\hline 14 & 28.0 & $<0.10$ & 8.0 & $\begin{array}{l}\text { Positive GAD and } \\
\text { insulin antibody }\end{array}$ \\
\hline
\end{tabular}

therefore recommended. Larger studies are needed to identify patients that are at increased risk of presenting with DKA and to better characterize the clinical course in this population.

Declaration of interest

The authors declare that there is no conflict of interest that could be perceived as prejudicing the impartiality of the research reported.

\section{Funding}

This research did not receive any specific grant from any funding agency in the public, commercial or not-for-profit sector.

\section{Patient consent}

We confirm that written informed consent has been obtained from the patient for publication of the submitted article.

Author contribution statement

Senhong Lee: study conception, literature review, drafting of manuscript, critical revision; Aparna Morgan: study conception, literature review, critical revision; Sonali Shah: study conception, critical revision; Peter $\mathrm{R}$ Ebeling: study conception, critical revision.

\section{References}

1 Godwin JL, Jaggi S, Sirisena I, Sharda P, Rao AD, Mehra R \& Veloski C. Nivolumab-induced autoimmune diabetes mellitus presenting as diabetic ketoacidosis in a patient with metastatic lung cancer. Journal for Immunotherapy of Cancer 20175 40. (https://doi. org/10.1186/s40425-017-0245-2)

2 Gauci ML, Laly P, Vidal-Treecan T, Baroudjian B, Gottlieb J, Madjlessi-Ezra N, Da Meda L, Madelaine-Chambrin I, Bagot M, Basset-Seguin N, et al. Autoimmune diabetes induced by PD-1 inhibitor - retrospective analysis and pathogenesis: a case report and literature review. Cancer Immunology, Immunotherapy 201766 1399-1410. (https://doi. org/10.1007/s00262-017-2033-8)

3 Hughes J, Vudattu N, Sznol M, Gettinger S, Kluger H, Lupsa B \& Herold KC. Precipitation of autoimmune diabetes with anti-PD-1 immunotherapy. Diabetes Care 201538 e55-e57. (https://doi. org/10.2337/dc14-2349)

4 Usui Y, Udagawa H, Matsumoto S, Imai K, Ishibashi M, Kirita K, Umemura S, Yoh K, Niho S, Ohashi K, et al. Association of serum anti-GAD antibody and HLA haplotypes with type 1 diabetes 
mellitus triggered by nivolumab in patients with non-small cell lung cancer. Journal of Thoracic Oncology 201712 e41-e43. (https://doi. org/10.1016/j.jtho.2016.12.015)

5 Abdul Aziz MH, Fernando IP, Lenkanpally A \& Fernando DJS. Diabetic ketoacidosis after treatment with pembrolizumab. Journal of Clinical and Translational Endocrinology: Case Reports 20175 4-5. (https://doi.org/10.1016/j.jecr.2017.05.002)
6 Cerf ME. Beta cell dysfunction and insulin resistance. Frontiers in Endocrinology 20134 1-12. (https://doi.org/10.3389/ fendo.2013.00037)

7 Fralick M, Scheeweiss S \& Patorno E. Risk of diabetic ketoacidosis after initiation of an SGLT2 inhibitor. New England Journal of Medicine 376 2300-2302. (https://doi.org/10.1056/ NEJMc1701990)

Received in final form 7 March 2018 Accepted 6 April 2018 